\title{
It's No Picnic: \\ Personal and Family Safety for Rural Social Workers
}

Authors: Rosemary Green, Raeleene Gregory and Robyn Mason

Key Words

\author{
Rural \\ Worker safety \\ Violence
}

Word length:

3950 excluding references

\section{Acknowledgements:}

Special thanks to Dr. Bob Lonne of QUT for assistance with the design of the questionnaire. 


\section{Background on Authors:}

\section{Key author:}

Associate Professor Rosemary Green

Highest qualification MSW

Head of School, Behavioural and Social Sciences and Humanities University of Ballarat

P.O. Box 663

Ballarat 3353.

Email r.green@ballarat.edu.au

As key author, implemented project with Robyn Mason, assisted with design of questionnaire, recruited and interviewed participants, and held regional forums. Drafted the article with co-authors, and contributed particularly the latter sections. Co-ordinated the drafting, editing and data analysis required and with co-authors refined and edited the article.

\section{Co authors:}

\section{Raeleene Gregory}

Highest Qualification:BSW

Previously Coordinator of Ballarat (Vic) Centre Against Sexual Assault. Currently post graduate student, University of Ballarat researching the adoption and management of privacy and boundary issues in rural welfare practice.

Email: rgregory@students.ballarat.edu.au

Contribution to this project:

Data analysis and drafting/editing of article.

Robyn Mason - co author

Highest Qualification:BSW

PhD Candidate, University of Melbourne School of Social Work, researching 'Rural Women's Services in Australia'

Most recent position: Coordinator, Ballarat Centre Against Sexual Assault, Victoria, Australia

\section{Email: robynm@netconnect.com.au}

Contribution to this project:

Implementation of initial project with Rosemary Green. Design of questionnaire, recruitment and follow-up of questionnaire participants. Involved in initial draft of article, especially introductory sections of article, and in later rewriting with co-authors 


\title{
It's No Picnic:
}

\section{Personal and Family Safety for Rural Social Workers}

Key Words: Rural Worker safety Violence

\begin{abstract}
This paper reports the key research findings related to personal and family safety of rural welfare and social workers, from a study conducted in rural Victoria, Australia. Significant findings included concerns about personal and family safety, frequency of episodes of work related violence and harassment, and the resultant impact on personal and family activities.
\end{abstract}

A range of useful strategies was identified to combat and cope with both the risk and experience of violence and harassment for the worker, and for their families. Workplaces, professional associations and educators need to recognize the impact of this occupational hazard, and respond with sensitivity to these issues, which have particular relevance for rural practitioners where anonymity and privacy are frequently compromised.

\section{Introduction}

The research reported in this article is part of a larger study of rural welfare and social workers in the Western Region of Victoria, which included an examination of factors relevant to rural practice, such as service delivery, management of dual and multiple 
roles, privacy and confidentiality. Investigation of work related harassment and violence was included in the study as our own experience, and the stories we heard informally from colleagues, indicated that this could be a significant issue for rural practitioners especially when working with statutory clients or perpetrators of violence and abuse. In these fields social work can be conceptualised as a form of social control rather than as a vehicle for empowerment. The social worker as an agent of control is not a new idea; proponents of the radical social work tradition have had concerns about it for many years (Ife 1997, p.33). Participants in our study often work with clients who feel powerless and threatened because of their situation. This can lead to behaviour that some may see as anti-social, directed at the workers because they are seen as representing the oppression that clients are suffering. (social workers)....work daily with vulnerable people: many of the factors which contribute towards that vulnerability- for example poverty, illness or family tensions- are factors which may well predispose those individuals towards violent or aggressive behaviour. In addition some clients may have a tendency towards temporary loss of control, for example because of alcohol or drug abuse.

Bibby (1994 p.19)

The challenge for social and welfare workers is to pursue empowerment while in a position of social control (Barber 1991, p.48).

\section{Understanding the Issues}

The risk of violence for workers in the human services has emerged as an occupational issue in the past two decades. On reviewing the literature, there are few studies which explore this aspect of practice. In Great Britain Bibby (1994) explored policy frameworks and practical strategies, such as assertiveness and interview 
techniques, to address violence in the workplace. In Canada Gair and Thorpe (1996) exposed the extent and nature of threats and actual physical violence experienced by workers, with up to $61 \%$ of workers reporting verbal abuse in one Canadian study. In Australia, in 1991 the increasing frequency of violence related to the distribution of emergency relief was recognized in a seminar organized by the Victorian Emergency Relief Project (Cheshire and Gourlay 1991). This was followed by a major study conducted by Puckett and Cleak (1994) which investigated 124 welfare and social workers working in 'high risk' settings in Melbourne, with results suggesting that almost one-third of participants in the study had been physically assaulted one or more times. In the same study, almost one-third of respondents, when asked how many times they had experienced verbal abuse, could not say how many, 'because of the frequency with which such abusive episodes occurred’ (Puckett and Cleak 1994, p. 4-5).

Horejsi and Garthwait's (1994) Amercian study identified that threats are often directed against the worker's family. Fifteen of the 166 child protection workers surveyed reported threats of violence directed at their family members. Gair and Thorpe (1996) found that five of the 89 respondents had their families threatened and a quarter of graduates surveyed had seen other workers experiencing violence.

Issues for individual rural practitioners include managing high visibility, informality, self-disclosure, trust and mistrust of professionals, dual and multiple roles, and blurring of professional and personal boundaries. (Briskman and Lynn 1999; Cheers 1998; Wilson-Barrett and Dollard 2000). High visibility, and the consequent lack of anonymity and privacy, for rural social workers and their families, has both positive 
and negative elements. For some, high visibility can lead to concerns about personal and family safety, particularly when working in fields such as family conflict and violence, child protection or the criminal justice system. Where urban workers may be able to maintain anonymity by living in different suburbs to those in which they work, rural social workers can be highly visible as they often must live, socialize and work within the same community.

Few studies have concentrated on issues of safety for rural social workers and their families. Horejsi and Garthwait’s (1994) findings indicated that threats, violence and verbal abuse were frequently experienced related to work roles in child protection. One third of their participants reported being frightened on the job at least monthly. Verbal abuse occurred up to several times weekly for about one quarter of the participants. They identified that threats were also made against workers' property and their family members. Supervisors responding in this study expressed concern about workers becoming desensitized:

...Many workers get so accustomed to threats and angry parents that they tend to underestimate or disregard the danger they really face”

Horejsi and Garthwait (1994, p.77)

There is very little Australian rural social work research about work related violence and harassment. Studies by nurses have indicated that there is a culture of nonreporting, denial and minimization of the importance of such episodes both by nurses and management (Fisher, Bradshaw, Currie, Koltz, Reid Serle, \& Smith 1996, Kelly 1998). 
The literature suggests that when nurses do report violent incidents, their employer and colleagues frequently respond by identifiying the individual's nursing and interpersonal skills, or lack of these, as somehow having caused or contributed to the problem.'

Fisher et al (1996, p.191)

This ‘blame the victim' approach is not uncommon in other professions, or indeed in the general community. Victims of violence are often made to feel responsible for the violence perpetrated on them. Outcomes of violence may include post-traumatic stress disorder (Kelly 1997), burnout, and vicarious or secondary traumatisation (Coholic \& Blackford, 1999, Kelly 1997, Sexton 1999) and these may be exacerbated by poor responses from colleagues and employers. The National Health and Medical Research Council (NHMRC) has recently developed a draft manual for health care workers, managers and employing organisations to assist workers in rural and remote communities to better manage episodes of violence. This manual considers a wide range of potentially violent situations, and includes examples of violence being directed against the health professional. (NHMRC 2002).

\section{Methodology}

The study examined issues for rural welfare and social workers in Western Victoria working in fields of practice identified by the researchers as 'controversial or conflictual'. These fields of practice included those where workers had statutory responsibilities such as corrections, child protection or mental health; or controversial fields that may threaten existing community views about women and children for 
example family violence and sexual assault. A University of Ballarat grant and a reference group of key staff from major regional organisations supported the research. The practitioner's experiences of harassment and violence arising from their work role were a key subset of this broader study. Participants included those with mandatory responsibilities and working in fields where conflicts frequently arise. The study utilized three methods of gathering data: a formal self-report questionnaire; informal, private interviews; and publicly advertised forums. While the sample size is small, this methodology, being both quantitative and qualitative, assists in analysing themes, narratives and experiences (Denscombe 1998, Lincoln and Guba 2000).

Questionnaires were widely distributed and completed by twenty-three participants. As would be expected in social work and welfare service delivery where the majority of workers are women, more women responded than men (19 women and 4 men). The participants represented a range of agencies, experience, qualifications and geographic locations. To reflect diversity of experience, age, agency focus, geographic location and size of community and agency, six were selected for interview. Those interviewed comprised one man and five women selected purposively to provide the study with variety of experience and background including rurality, length of practice, type of role and agency.

Following the completion and part analysis of the questionnaires, three forums were held in different parts of the region. These were widely advertised. Attendance at the forums totaled more than forty participants, of whom one or two had completed questionnaires. 


\section{The Participants}

Questionnaire respondents comprised mostly experienced practitioners, who resided in relatively small communities. Most had formal qualifications in social work or welfare, with the remainder being either qualified nurses or holding other tertiary qualifications.

Of the twenty-three questionnaire respondents, nine worked in a non-government organisation, twelve worked for a State Government agency or instrumentality, one in local government, and one recorded "other" in response to this question. Sixteen were in permanent positions. While four had been in their current position less than twelve months, eight had held their position between one and five years and ten had held their current position for over five years. Of the thirteen participants living in communities of fewer than 60,000 people, six lived in communities less than 10,000, three in townships of $10-19,000$, four between $39,000-60,000$ and nine did not specify. Three quarters had lived in the community for longer than seven years.

For fourteen participants visibility related to their work role was rated as very high, and while five felt very professionally isolated, nine reported feeling not professionally isolated at all and nine only a little or moderately isolated. Three quarters believed residing in a rural area had more advantages than disadvantages.

Overall, most respondents appeared to see living and working in rural practice as rewarding and suiting their lifestyle at present. While high visibility related to their work role existed, most felt comfortable with that. Overall professional isolation did not seem to be a major factor. 


\section{Definitions}

For the purposes of this study, the following definitions were used:

Work related violence is defined as any incident in which an employee is abused, threatened or assaulted by a member of the public in circumstances arising out of the course of their work, whether or not they are on duty. It includes behaviour, which produces damaging or hurtful effects, physically or emotionally.

Harassment is defined as any form of behaviour that a person does not want, and does not return, and that offends, humiliates or intimidates them.

\section{Experiences relating to personal safety}

Five of the questionnaire respondents estimated that they experienced violence on a daily or weekly basis, with slightly more (6) reporting episodes of harassment being daily or weekly. Five respondents reported never experiencing work place harassment while only three reported never having experienced episodes of violence. Harassment was experienced less often, but this finding may be explained by blurring of our definitions. Violence was infrequent, occurring less than once a month for 10 workers. (Refer Figure 1)

One participant described the reality of rural practice:

I feel that living and working in a rural community is full of risks...the nature of my work is that there are always risks.... People don't always understand 
my work objectives and may seek to exact some kind of payback for real or imagined actions I have taken in my professional capacity.”

Insert Figure 1 and Table 2

Violence and harassment occurred mainly in the workplace. Of the twenty questionnaire respondents who experienced violence, these episodes occurred mainly in the office, the telephone, and in the client's home. Violent episodes were reported to have occurred outside the workplace, in public or in the client's home for five of our sample. In fifteen cases, people known by the worker perpetrated this violence. Analysis indicated that harassment followed a similar pattern. (Refer Figure 2)

Insert Figure 2 and Table 2

Participants in forums referred to client anger, intimidation and threats as examples of violence. Sixteen questionnaire respondents referred to intimidation and verbal abuse, four had experienced physical violence, and three had received threats in person or by telephone after hours.

At the forum, participants spoke of being fearful when attending families living in remote locations, of having guns pointed in their direction, and of assaults such as punching, shoving and hitting. These events had occurred to several of the forum participants. Although they often attended home visits "in pairs" participants felt that 
this afforded them little real protection. While some situations were obviously risky, and in these cases office visits were arranged, participants disclosed episodes where they were 'taken by surprise' by aggressive and abusive situations. Participants in forums spoke of being verbally abused or otherwise threatened by clients or partners or former partners of clients in supermarkets, in the street or at the office.

Examples of harassment included verbal abuse, invasion of privacy at social events and in public places, episodes of stalking, and excessive phone contact. Theft of personal belongings, sexual references and unwanted romantic attention was reported in a few instances, as was malicious gossip and complaints.

Participants talked about the risks involved in solo home visits, transporting clients, and using personal vehicles. The lack of effective agency equipment such as radios, mobile phones, tracking systems, protocols and procedures relating to worker safety were identified as of concern. The reporting of episodes, and any resultant action, varied. Similar to comments made in studies conducted by nurses (Fisher, Bradshaw, Currie, Koltz, Reid Serle, \& Smith 1996, Kelly 1998), examples were given where violence was minimised, laughed away, or seen to be just part and parcel of the job by colleagues, the worker themselves and management. One of our participants commented:

My work mostly relates to a client group where violence and harassment is pretty much part of the work... mostly emotional and mental abuse and verbal harassment. While there is a threat of physical violence, this is far less 
threatening to me than these other forms of violence which I face on a daily basis”.

\section{Strategies for dealing with personal safety issues}

Workers reported a range of strategies for dealing with personal safety issues. These included the use of security pagers, duress alarms and mobile phones. However, at the forums it was highlighted by many participants that in rural areas mobile phones are unreliable, and do not provide real security for workers. Agency practices of recording the location of rural workers when on home visits varied, with many lone workers having little or no means of ensuring someone knew where they were going. Lone workers raised safety and confidentiality as a concern. They felt it would compromise confidentiality to advise the address of their client and other details relating to home visits to another person or agency, even though this may give them some personal security. Some agencies had a very specific protocol about time at home visits, and check up phone calls if a worker had not reported into the office within a specified time period.

Many participants at the forums and six of the questionnaire respondents reported that they were very clear and careful about highlighting boundaries, maintaining a professional distance from clients, and working within strict agency 'guidelines' so that the client would be less likely to personalise the actions taken by the worker.

Avoidance strategies were frequently mentioned. These included social workers being constantly aware of their proximity to clients, vigilance in public areas. Social workers frequently chose not to shop in the local area, or chose to shop when fewer 
clients were likely to be shopping. Some avoided taking family members, particularly children, with them in public. Avoiding social events in public spaces such as hotels and restaurants and preferring to socialize at home or in friends' homes were frequently cited. Using silent home phone numbers, using a different name at work, keeping the personal car away from the office, and avoiding solo home visits wherever possible were other strategies utilised.

One worker had recently taken further steps to relieve the stress she experienced. She reported: 'I have recently moved from the local community where I work as the stress of living and working in the same place has become too great'. She had arranged with her agency for her not to directly work with people in her new locale, but had already had an episode where a neighbour had sought her assistance "informally and out of hours” to escape an episode of family violence. One interviewee talked at length about her discomfort when clients moved into the house next door.

Some people identified that it was useful to have "local” knowledge as it enabled them to identify and avoid potential issues. Work practices that emphasised mutual respect and 'collaborative, working together approaches' to resolve issues were mentioned as good preventative strategies, but it was also acknowledged this was not a general solution in all situations. One worker advised: 'Always treat people with respect and courtesy, they could end up being your neighbour'. Others stated that clearly identifying boundaries and having work protocols assisted in managing potential workplace harassment and violence. Good supervision, support and opportunities to debrief were considered very important to managing stressful episodes. 
These strategies had become part of the workers' repertoire and incorporated into their daily routine. For many rural welfare and social workers in the study, these measures were 'just part of life', but as researchers we were surprised at the level of compromise made by many workers in their personal lives as a direct result of their work role.

\section{Concerns about family safety}

Just under one quarter of respondents to the questionnaire reported that their work raised issues of family safety and close to one third had significant concerns about family safety. (Refer Figure 3)

Insert Figure 3 and Table 3

Experiences reported included phone calls to family members, which were threatening, general threats about 'getting even', and concerns for children, particularly where sexual offenders on probation or parole were in the worker's caseload. In the interviews, the forums and questionnaire analysis, it appeared that women workers with school age or adolescent children were often 'hypervigilant' about their children's whereabouts and their children's social contacts, and were highly concerned for their safety. However, the lack of male participants has not allowed us to further analyse gender as a critical factor. 


\section{Strategies to deal with family safety issues}

In terms of children's safety, some workers said that their children had mobile phones so as to inform parents of their whereabouts although the problem of reception in rural areas remains. At times children were told about risks to them posed by people in the community known to the worker through her professional role. Heightened awareness about children's safety was a factor for several workers responding to the questionnaire and in the forums. One told us: 'I never allow my 15 year old daughter to be alone in public and always know her whereabouts'. Others reported that they exercised no more than the usual parenting vigilance, and that there was a safety inherent in rural communities: 'We all look out for each other's kids'. Others deliberately educated members of their family about the need for additional care related to privacy, safety and the work role. Some reported warning their family members of suspected dangers, and had provided instructions about how to deal with client or client-related phonecalls or visits.

Another strategy involved women choosing to use a different name, such as their maiden name at work, to avoid being identified elsewhere. For many rural workers in smaller communities this was seen as ineffectual as residents knew them and their families. For some this was positive:

Being a rural person, and a member of this community gives me an 'edge' in some respects. I don't have to waste time building rapport, I am already known, and people trust me, particularly the elderly, as I understand the history and culture of the area. My conservative family background probably helps too. " 
This interviewee had few fears for her family, as her adult children were 'big men and could take care of themselves'. Rather, her concerns were related to community safety issues, for example, when she knew of repeat child sex offenders being released from prison and settling in the town.

Some participants stated they did not talk about work at home or give details of the professional role, as a means of protecting family members from worry about the worker. The reverse was also used as a strategy, where the worker was very circumspect about her family connections, and took care that there was no acknowledgement of her family life in the workplace. Three participants commented as follows:

My family are totally unaware of my roles.

I deliberately do not have photos in my office.

I don't want to be seen with my grandchildren in this community... for their own protection it is better if they are not identified as belonging to me...nor do I have their drawings or other items around the office.

Several workers refrained from displaying pictures of their family or children's drawings. However, for some workers this was not a concern. They argued that such activities made them more ‘approachable and human’ and provided a normalising setting for clients, demonstrating trust and respect. 


\section{Conclusion}

This study reflects the many challenges faced by rural social workers in terms of personal and family safety, and confirms the findings of previous studies. There is some evidence that in rural areas the percentage of social workers experiencing episodes of violence and harassment is even higher than that reported by Gair and Thorpe(1996) and Puckett and Cleak (1994). The concern for family safety is significant with a higher percentage of participants reporting concern, in excess of the findings reprted by Horejsi and Garthwait(1994).As this study was small, further investigation is warranted, however reports of violence and harassment seem significantly high, and there is some evidence that, like nurses, this is taken for granted as part of the role, minimized, and its impact hidden. Its role in burnout, job dissatisfaction and vicarious trauma should also be further investigated. A study of gender and its impact on violence and harassment would be useful, as this study was too small, and lacked enough male participants to draw any real conclusions about gender. The occurrence of hypervigilance as a result of workplace violence and harassment, in both urban and rural workers in contentious fields of practice, is another area worthy of further study.

Individual workers have developed a range of strategies, appropriate to their particular professional approach, personal values and experience. Strategies used by some are in direct opposition to strategies developed by others, such as displaying family photos at the office or disclosing family background. These different perspectives do not alter the findings that harassment and abuse related to work roles are significant in rural areas and that they impact on workers and their families. 
Educators and employers must take into account these safety factors when establishing services and recruiting staff in rural areas. There are also implications for professional training and for rural communities.

First, individuals who want to work and thrive in contentious rural social welfare positions need to be resilient, with good coping strategies equipping them to face difficult personal and family challenges arising from their work. Professionals who have experience on the ground, such as those in our study, would be useful role models and mentors for newly graduated or newly arrived rural workers.

Secondly, rural service providers need to be aware that these challenges exist and pose a threat to the wellbeing and productivity of their workforce. Protocols and policies that protect workers and acknowledge the risks they face, should be standard items in the policy manuals of agencies and included in induction and in-service education programs. Such approaches should not tolerate threats, abuse or violence against their staff. Workers should have an expectation that the agency's duty of care to staff is informed by this knowledge. When recruiting new staff, the challenges of rural practice should be outlined to interested applicants, not in an alarmist way, but to alert recruits to the reality of rural professional life. Successful strategies used by experienced staff should be recommended to new workers early in their role, with a mentoring system as a possible tool. Should episodes of violence occur, access to counselling for workers, their families and especially their children, should be provided quickly. There must be appropriate protocols to manage these incidents without minimising them or their impact, including reporting threats to police where necessary. Ongoing education and training for staff in how to minimise their 
vulnerability to episodes of violence and abuse should be provided. Protocols should be established with other key agencies regarding management of volatile situations involving clients. Effective supervision and opportunities to debrief must be provided on a regular basis to address these concerns.

Thirdly, professional associations and higher education institutions need to be active in disseminating research findings about the challenges of rural practice in professional development and teaching roles. Students from rural areas will often return to their home town or region to work. Others who do not envisage working in rural areas may take up rural positions during their working life. Students need to be aware of the lessons learnt by those who have gone before them, and examples of rural practice issues should be included in the curriculum.

Finally, we should reconsider the theory and practice of working in contentious and conflictual fields. It must be recognized that social and welfare workers often engage in work where they excert socially sanctioned use of power, and where behaviour is often determined by someone else as deviant or unacceptable. We should further explore the nature and implications of this work for practitioners in both rural and urban settings.

$\mathrm{e}$

While this study has focused on the experiences of rural welfare and social workers, issues of personal and family safety constitute significant career considerations for all health and welfare personnel and demand our attention. 


\section{References}

BARBER, J. G. (1991) Beyond Casework, Macmillan, London.

BIBBY,P. (ED) (1994) Personal Safety for Social Workers Aldershot, England.

BRISKMAN, L. \& LYNN, M. (EDS). (1999), Challenging Rural Practice, Deakin University Press, Geelong.

CHEERS, B. (1998), Welfare Bushed: Social Care in Rural Australia, Ashgate, Birmingham.

CHESHIRE, J. \& GOURLAY,M. (EDS) (1991) 'Violence and emergency relief:its getting worse, what to do?' VCOSS Papers No 8. Victorian Council of Social Services, Victoria.

COHOLIC, D.A.\& BLACKFORD, K.A. (1999), 'Vicarious Trauma in Serious Assault Workers: Challenges of a Northern Location', in Promoting Inclusion Redressing Exclusion, Joint AASW, IFSW, APASWE, AASWWE Conference Proceedings, vol 1 pp 242 - 248, Australian Association of Social Workers, Brisbane.

DENSCOMBE, M. (1998), The Good Research Guide: for small research projects, Open University Press, Buckingham, England.

FISHER, J., BRADSHAW, J., CURRIE, B.A., KOLTZ, J., REID SERLE, K. \& SMITH, J. (1996), 'Violence and Remote Area Nursing', Australian Journal of Rural Health, Vol 4, No 3, pp 190 - 199. 
GAIR, S. \& THORPE, R. (1996), 'Helpers as Victims: Assaults on Women in Social Welfare Practice', Chapter 7, in Thorpe, R. and Irwin, J. (Eds) Women and Violence: Working for Change, Hale and Ironmonger, Sydney.

HOREJSI, C. \& GARTHWAIT, C. (1994), ‘A Survey of Threats and Violence Directed against Child Protection Workers in a Rural State', Child Welfare, vol 73, no 2.

IFE, J. (1997) Rethinking Social Work: Towards Critical Practice, Longman, Melbourne.

KELLY, K. (1998), 'Responding to job related trauma in remote areas: only fools would rush in', in $4^{\text {th }}$ Biennial Australian Rural and Remote Health Scientific Conference, Toowoomba, Queensland 1998 Proceedings, National Rural Health Alliance Inc, Rural and Remote Health Papers, 1991 - 2001, CD Rom.

LINCOLN, Y.S. \& GUBA, E.G. (2000), 'The only generalisation is - there is no generalisation', Chapter 2, pp 27 - 44, in Gomm, R., Hammersley, M. \& Foster, P., (Eds) Case Study Method, Sage, Great Britain.

NATIONAL HEALTH \& MEDICAL RESEARCH COUNCIL, (2002), 'When its right in front of you- Assisting health care workers in rural and remote Australia to manage episodes of violence', Consultation Draft http://www.health.gov.au/nhmrc/advice/consultation.htm 
PUCKETT, T.C. \& CLEAK, H. (1994), ‘Caution - Helping may be Hazardous:

Client abuse, threats and assaults’, Australian Social Work, vol 47.1, pp 3-9.

SEXTON, L. (1999), ' Vicarious Traumatisation of Counsellors and Effects on their Workplaces’, British Journal of Guidance and Counselling, vol 27, issue 3.

WILSON-BARRETT, E. \& DOLLARD, M. (2000), 'Dual Relationship Dilemmas’, in Munn, P \& Farrin, J., (eds), Constructing Alliances Across Rural Communities: Proceedings of the $4^{\text {th }}$ National Regional Australia Conference, vol 2, Rural Psychology, pp 9-15, South Australian Library Publications, University of South Australia, Adelaide. 
INSERT FIGURE 1

\begin{tabular}{|l|c|c|}
\hline & $\begin{array}{c}\text { Frequency of } \\
\text { work-related } \\
\text { violence }\end{array}$ & $\begin{array}{c}\text { Frequency of work- } \\
\text { related } \\
\text { harrassment }\end{array}$ \\
\hline count & count \\
\hline $\begin{array}{l}\text { Daily/Every couple of } \\
\text { days }\end{array}$ & 3 & 4 \\
\hline Weekly & 2 & 2 \\
\hline $\begin{array}{l}\text { Every couple of } \\
\text { weeks }\end{array}$ & 2 & 1 \\
\hline Monthly & 3 & 2 \\
\hline $\begin{array}{l}\text { Less than once a } \\
\text { month }\end{array}$ & 10 & 8 \\
\hline Never & 3 & 5 \\
\hline Total & 23 & 22 \\
\hline
\end{tabular}

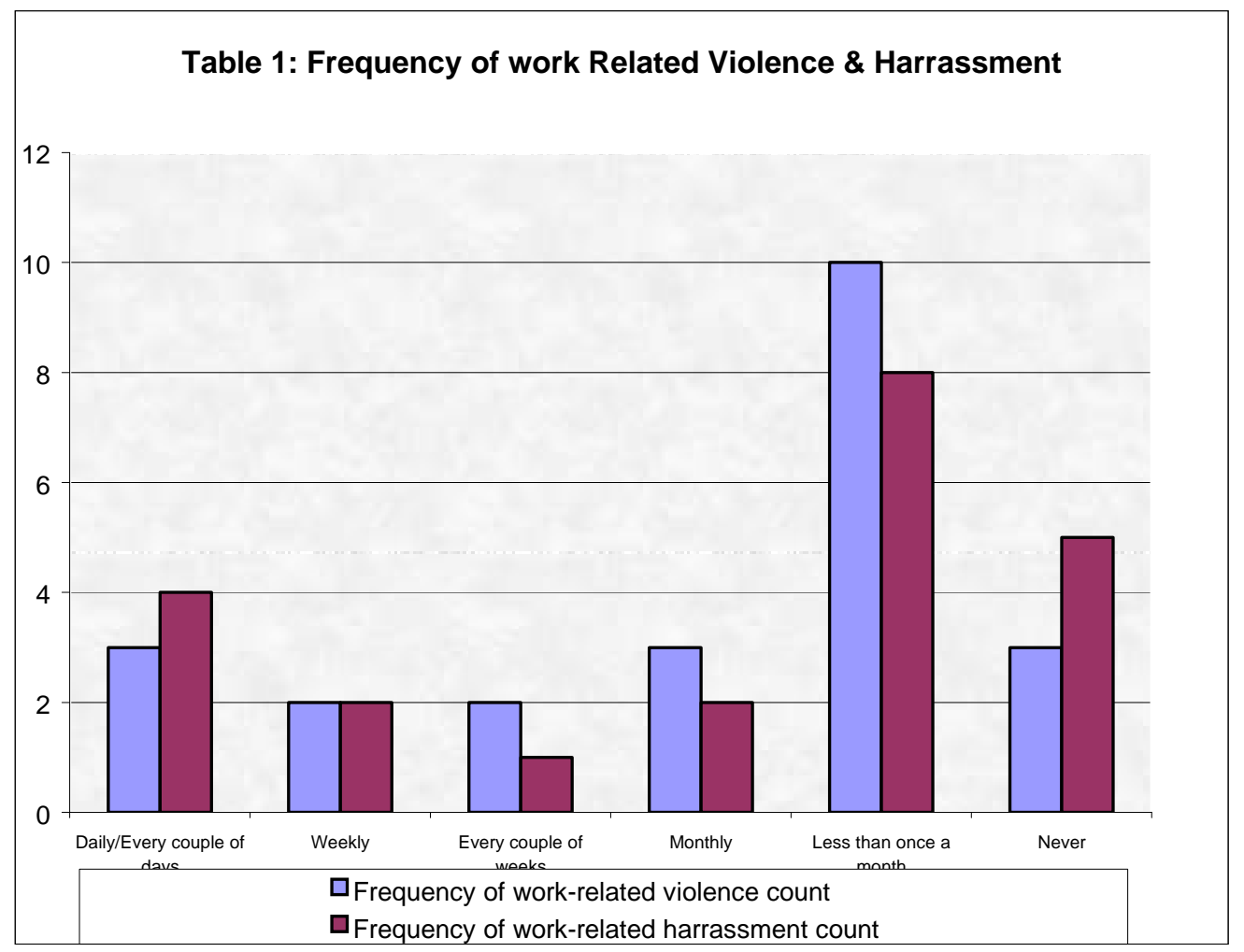


INSERT FIGURE 2

\begin{tabular}{|l|c|c|}
\hline & $\begin{array}{c}\text { Work-related } \\
\text { violence }\end{array}$ & $\begin{array}{c}\text { Work-related } \\
\text { harrassment }\end{array}$ \\
\hline & count & count \\
\hline In the workplace & 17 & 14 \\
\hline In tclient's home & 7 & 5 \\
\hline In the car & 3 & 1 \\
\hline In public places & 3 & 3 \\
\hline On telephone & 9 & 9 \\
\hline $\begin{array}{l}\text { In the mail or } \\
\text { electronically }\end{array}$ & 1 & 0 \\
\hline In other places & 2 & 1 \\
\hline Total & 42 & 33 \\
\hline
\end{tabular}

Table 2: Where Work Related Violence \& Harrassment Occurs

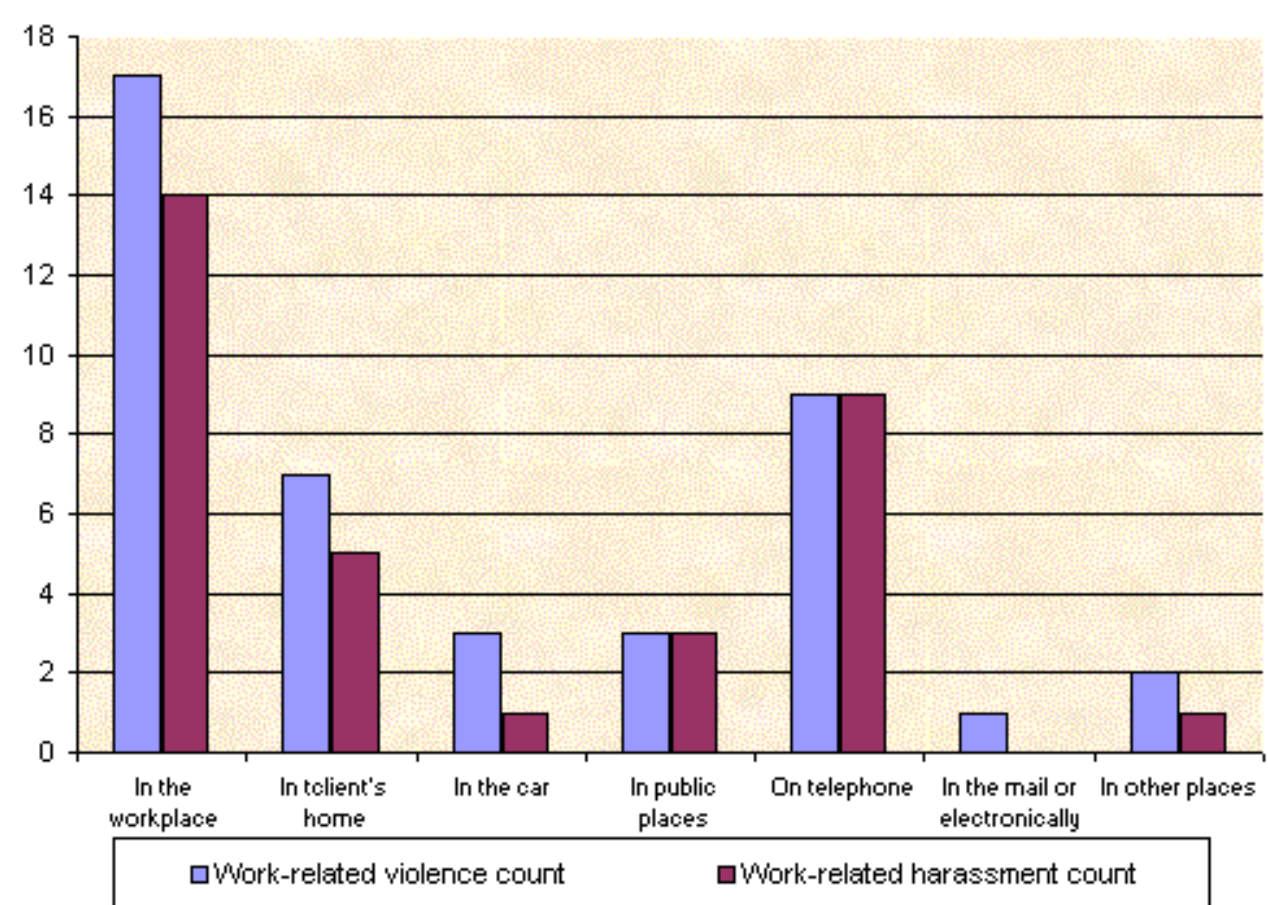


INSERT FIGURE 3

\begin{tabular}{|l|c|}
\hline & $\begin{array}{c}\text { Concern for } \\
\text { family safety }\end{array}$ \\
\hline Not at all & 10 \\
\hline A little & 1 \\
\hline A moderate amount & 5 \\
\hline A good deal & 7 \\
\hline Total & 23 \\
\hline
\end{tabular}

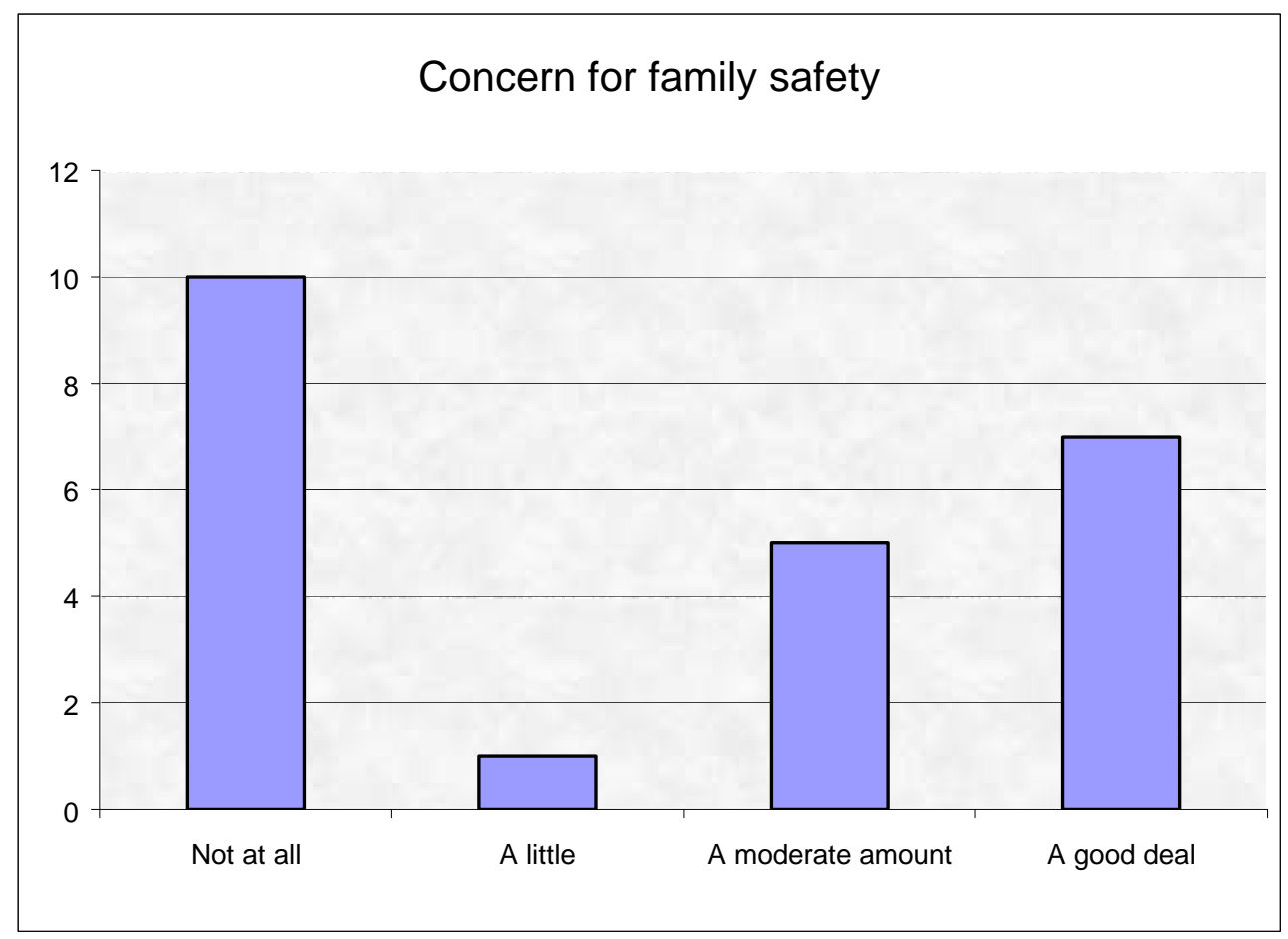

